\title{
Different Approaches on Various Cases of Tracheal Stenosis
}

\author{
Sawang Saenghirunvattana, Vitoon Pitiguagool, Chokchai Suwanakijboriharn, \\ Pakorn Pupipat, Bhudsadee Saenghirunvattana, Maria Christina Gonzales, \\ Kritsana Sutthisri, Chitchamai Siangproh, Wannipa Kodkaew, Assarin Inkum, \\ Vimonsiri Matitopanum
}

Bangkok Hospital Medical Center, Bangkok Hospital Group, Bangkok, Thailand

Email: Sawang.Sa@bangkokhospital.com

Received 26 May 2014; revised 25 June 2014; accepted 24 July 2014

Copyright (C) 2014 by authors and Scientific Research Publishing Inc.

This work is licensed under the Creative Commons Attribution International License (CC BY).

http://creativecommons.org/licenses/by/4.0/

(c) (i) Open Access

\begin{abstract}
Narrowing of the airway caused by different diseases is a serious condition manifesting varying signs and symptoms. Immediate attention and treatment must be performed as this is a lifethreatening condition. In the past decade, there has been massive advancement on the management of airway stenosis. Some of these are stent placement, tracheal reconstruction and tumor debulking. This article focuses on 5 different cases with distinct strategies in conducting treatment and management of airway stenosis.
\end{abstract}

\section{Keywords}

Central Airway, Tracheal Stenosis, Stent Placement, Tracheal Resection

\section{Introduction}

Airway stenosis is the partial or complete narrowing of the central airway passages. This may involve the subglottic, trachea-esophageal, broncho-esophageal or tracheo-broncho-esophageal area. The disease may be caused by focal inflammation or trauma (prolonged tracheostomy or intubation), systemic inflammation, infectious disease (tuberculosis), or malignancy (primary or metastatic). It is a potentially life-threatening condition with varying signs and symptoms. Patients frequently do not recognize any symptom until at least $50 \%$ of the luminal diameter is compromised because of the distensible character of esophagus. This is explaining the late presentation and/or prognosis associated with the disease [1]. Patients with this disease not only have a limited life expectancy but also may suffer from debilitating complications. Management of this condition requires immediate attention, thorough physical examination, diagnostic tests (CT scan, tomography), accurate diagnosis and ulti-

How to cite this paper: Saenghirunvattana, S., Pitiguagool, V., Suwanakijboriharn, C., Pupipat, P., Saenghirunvattana, B., Gonzales, M.C., Sutthisri, K., Siangproh, C., Kodkaew, W., Inkum, A. and Matitopanum, V. (2014) Different Approaches on Various Cases of Tracheal Stenosis. Open Journal of Respiratory Diseases, 4, 90-100. 
mate definitive treatment. Treatment includes systemic therapy and appropriate technique specific for each case. Though there are many types, causes and management of tracheal stenosis, this article describes 5 cases of adult airway stenosis (3 malignant and 2 benign) and the team's unique approach in managing both malignant and benign conditions.

\section{Aims and Objectives}

We performed this study to analyze the different techniques used in handling 5 patients with different pathology/ or causes of airway stenosis.

\section{Method}

Between September 2012 and November 2013, we identified 5 patients diagnosed of central airway narrowing. All cases had symptomatic airway stenosis. We analyzed each case study, considered the pathology of the disease, treatment, prognosis, and improvement in quality of life.

\section{Case Reports}

\subsection{Case Report \# 1}

A case of a 46-year-old male patient diagnosed of Lung Cancer stage III. He was referred to our medical facility 9 months post right upper lobectomy and following external irradiation and chemotherapy. The cytopathology report from the lobectomy revealed large cell undifferentiated carcinoma, suggestive of adenocarcinoma with $90 \%$ tumor necrosis, size $=7.0 \times 6.0 \times 4.2 \mathrm{~cm}$.

Physical examination revealed coughing for 2 months, progressive difficulty and shortness of breathing for 2 weeks, orthopnea, rhonchi upon auscultation of the lung and pain when in right lateral position. No cardiac problems were noted. Neck, chest and abdominal Computerized Tomography (CT) scan presented a demonstrable consolidative mass with heterogeneous enhancement, central necroses, haemorrhage and lobulated contour; measuring about $82 \times 48 \mathrm{~mm}$ in the right lung apex, prevertebral and paratracheal regions. This mass extends to the pleurae, periphery, adjacent upper thoracic vertebrae, upper trachea \& upper esophagus. The mass causes pressure that affects the upper trachea and upper esophagus which results to upper tracheal stenosis or obstruction of more than $80 \%$ - $90 \%$ (Figure 1 ).

The patient was advised to be admitted for bronchoscopic evaluation and insertion of a small endotracheal tube to establish patent airway. The direct visualisation of the location of the tumor was crucial to determine whether it is external or endobronchial in nature and in order to consider electrocautery in extraction of the mass or if stent placement was possible. A team of oncologists, anaesthesiologists, and cardiovascular surgeon were consulted on this scenario. The patient and relatives were well aware of the high risk of this procedure and possible complications that may occur such as complete airway obstruction, haemorrhage, infection, and mortality. The prognosis and better quality of life and alternatives were discussed and they acknowledged but decided to delay the treatment.

Two months after the initial consultation, after progression of symptoms, the patient decided to undergo the bronchoscopy and stent placement. Flexible bronchoscopy showed endobronchial mass nearly completely obstructing the main airway. Rigid bronchoscope was used for stent placement. Polyflex airway stent was successfully inserted and the patient did not manifest complications (Figure 2). On the succeeding months, the patient underwent brachytherapy for treatment. Three months post stent placement, the patient came back complaining of dysphagia. Positron Emission Tomography (PET) CT scan presented a continuous increase in size of the mass at the superior mediastinum which extended to right upper paramediastinal region and partially encased trachea, now measuring about $6.9 \times 5.2 \mathrm{~cm}$ at sternal notch level. The tracheal stent is in place from sternal notch to carinal level. The mass affects the proximal Superior Vena Cava. The superior extension of this mass which extended beyond the apical lung, involved scalene muscle and has compressed to right brachial plexus which also increased in size from $1.5 \mathrm{~cm}$ to $2.3 \mathrm{~cm}$. He was then admitted to undergo fiberoptic bronchoscopy and electrocautery to remove the tumor on both ends of the stent and suction mucus plug (Figure 2). Post procedure, he was improving symptomatically with the infusion of antibiotics and palliative radiation therapy to the affected part of the tumour mass. The patient continued receiving treatment until he expired 8 months post stent placement. 


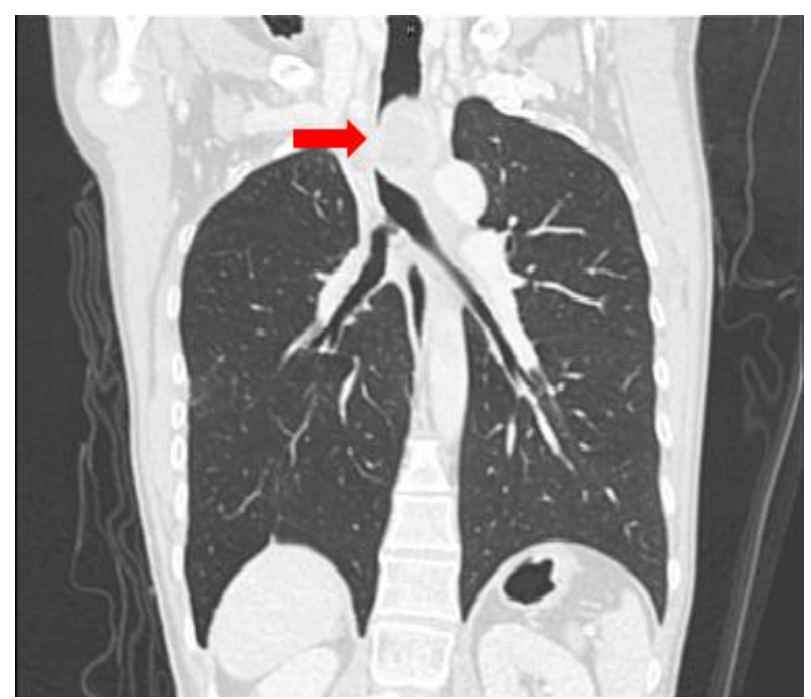

Figure 1. CT scan image demonstrating consolidative mass.

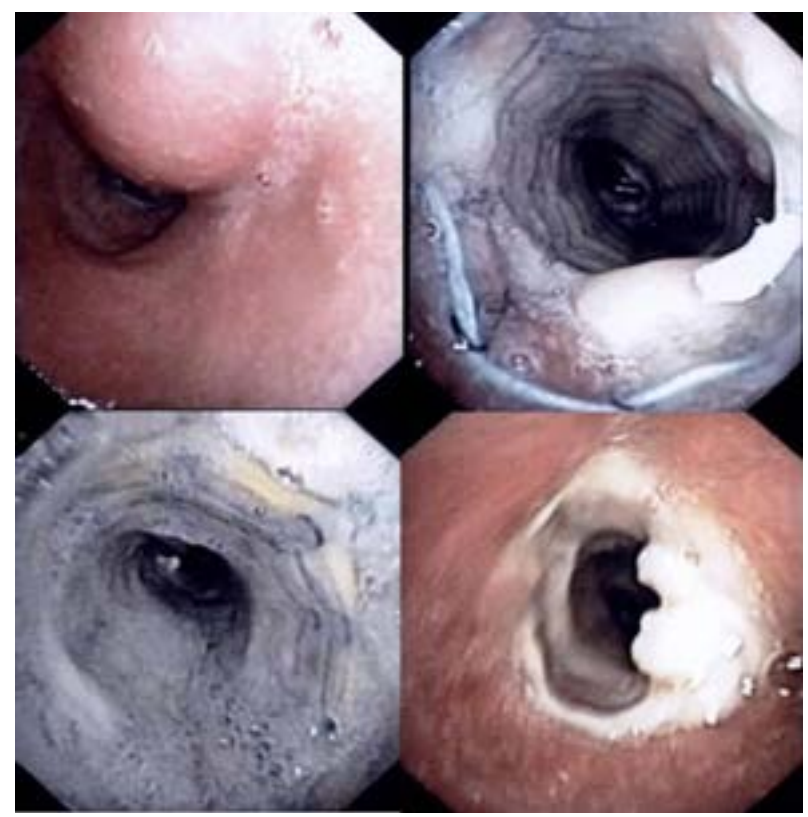

Figure 2. Shows (clockwise from upper right) part of the mass, the stent placement, mucous plug and tumor regrowth.

\subsection{Case Report \# 2}

The patient is a 46-year-old male who presented with progressive dyspnea for 4 months, hoarseness of voice and stridor for 2 months then fever, and cough with greenish secretions for 2 weeks. He was a previous smoker for 20 years but stopped 6 months prior to consultation. Physical examination revealed stridor upon auscultation. Chest CT scan revealed thickened trachea, more on the posterior wall about $5 \mathrm{~cm}$ in length from about lower T1 to lower T4 level, causing narrowed trachea (Figure 3 and Figure 4). Multiple pulmonary nodules at least 7 in the right and 5 in the left lung were noted. Metastasis was suggested and the largest nodule near right hilum may be primary carcinoma. Laboratory markers were significant for Carcinoembryonic Antigen (CEA): $5.49 \mathrm{ng} / \mathrm{ml}$, Hemoglobin: $11.4 \mathrm{~g} / \mathrm{dl}$ and White Blood Cell (WBC): $13.54 \times 10^{3} / \mathrm{mm}^{3}$. The patient was then intubated with the use of microlaryngeal tube no. 5 for the endotracheal tube no. 8 was unable to pass through. The plan was that once stable, he will undergo Fiber Optic Bronchoscopy (FOB) for tracheal examination, visualization, biopsy, 


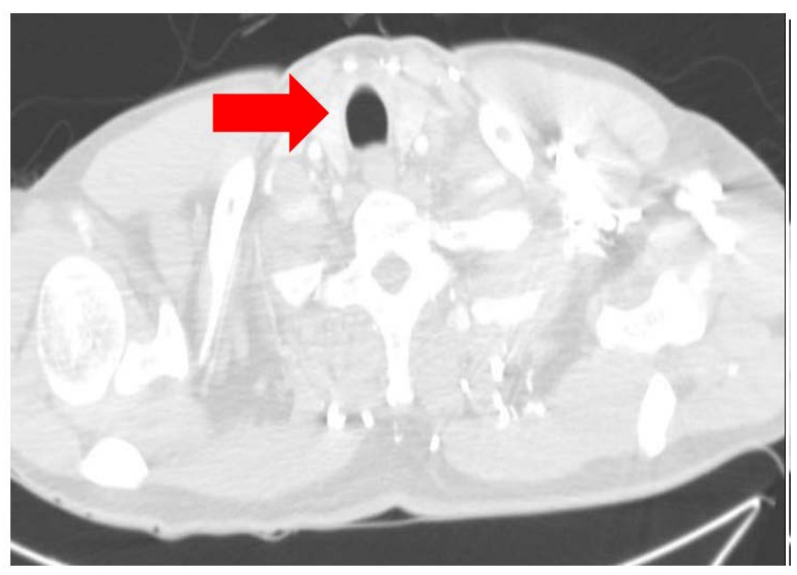

Figure 3. The normal upper trachea.

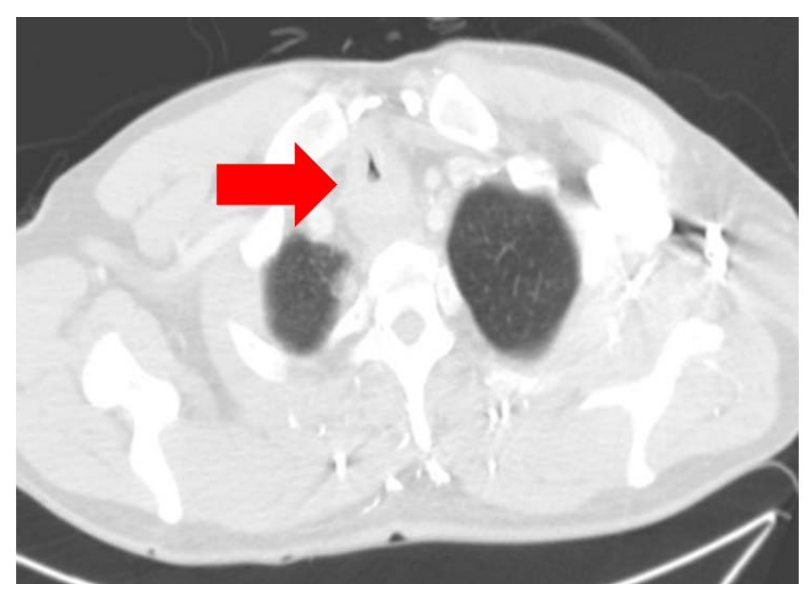

Figure 4. Stenosis at the mid portion of the trachea.

and electrocautery then stent placement with the use of rigid bronchoscope. The risk of hypoxia was greatly recognized. 2 days prior to the scheduled procedure, the patient exhibited hemoptysis. Bedside FOB was done to investigate and ceased the cause of bleeding by electrocautery. The following day the patient exhibited bradycardia because of sedative effects of anesthesia but was controlled and managed properly. Once stable he underwent FOB which revealed tracheal stenosis due to thickened endotracheal wall. Stent placement was done using rigid bronchoscopy. Polyflex airway stent was successfully inserted. Specimens were sent to the laboratory for cytology and revealed that tracheal tissue was positive for adenoid cystic carcinoma (Figure 5). The patient was able to recover and he was advised to undergo brachytherapy instead of chemotherapy or radiotherapy for this kind of tumor grows faster when stimulated as agreed during the tumor panel discussion.

\subsection{Case Report \# 3}

This is a case of a 69-year-old male patient diagnosed of esophageal cancer with metastatic brain tumor status post craniotomy for removal of tumor. He was referred for co-management of tracheal invasion. He was a known smoker for 10 years. He first complained of difficulty of breathing 6 months prior to consultation. Physical assessment revealed cough with whitish secretions, secretory sounds upon auscultation, dysphagia, abdominal discomfort, and episodes of fever. The neck and chest CT scan revealed a mass that invades the posterolateral aspect of upper intrathoracic trachea, causing intraluminal narrowing (6 mm in AP dimension) which also invades posterior part of the right lobe of thyroid gland. A new lobulated contour subpleural nodule was also seen at posterobasal segment of right lower lobe, size $=2.1 \mathrm{~cm}$. Few millimiters subpleural nodules were seen in posterior segment of right upper lobe, apicoposterior segment of left upper lobe, superior segment of left lower lobe, and anterior basal segment of left lower lobe (Figure 6). Since the patient has been battling with metastatic 


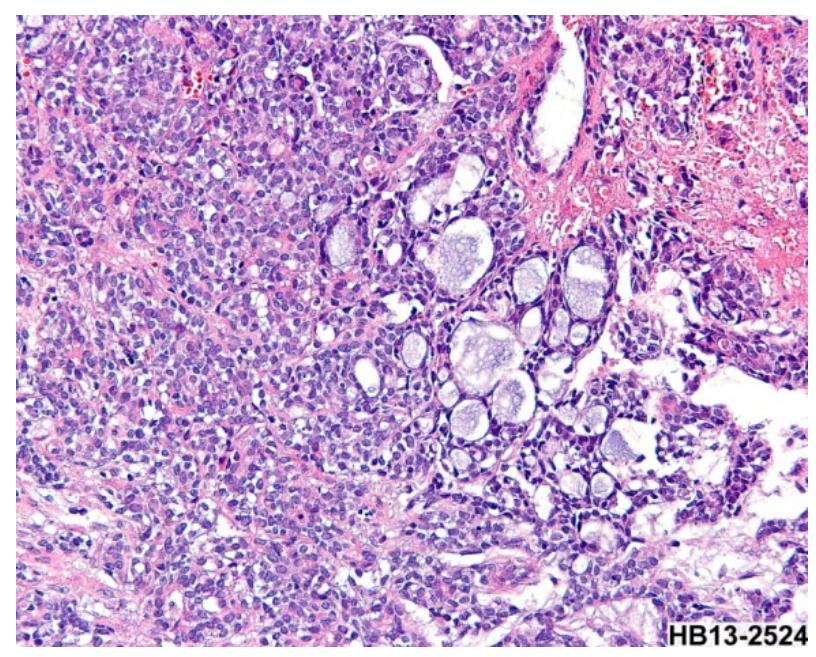

Figure 5. Adenoid cystic carcinoma as described in case \# 2.

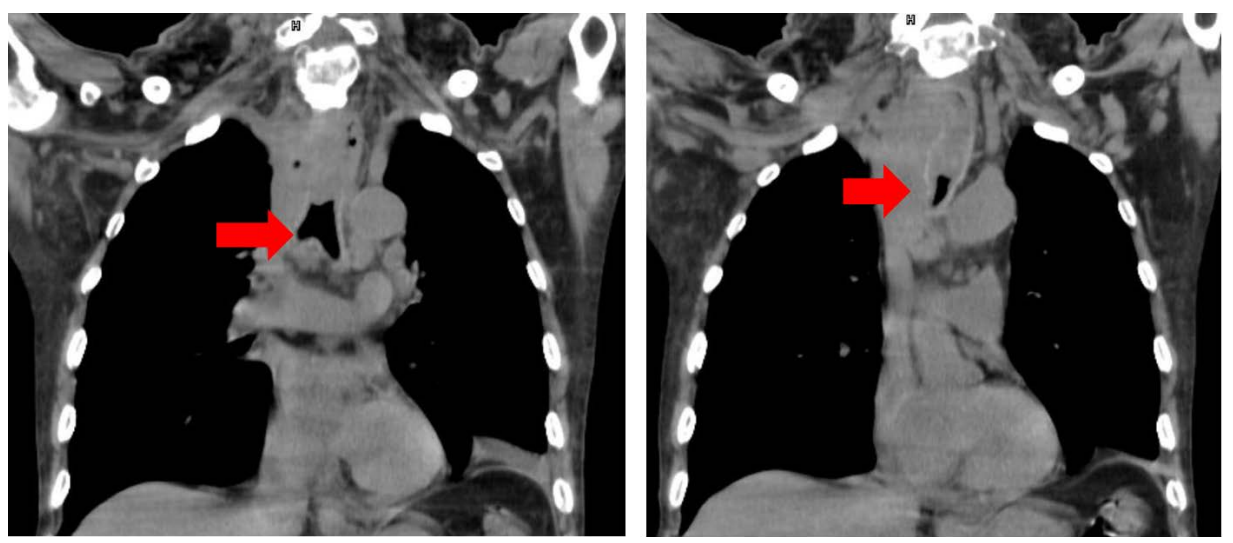

Figure 6. CT scan image revealing a mass that invades the upper intrathoracic trachea.

brain cancer, palliative treatment of stent placement was offered. Upon admission, the patient underwent chest CT scan and the result revealed diffuse centrilobular nodules with interlobular septal interstitial thickening, multiple significant mediastinal lymphadenopathy and segmental esophageal mass (primary carcinoma); measuring about $6 \mathrm{~cm}$ in length at level of cervico-thoracic junction. He was then scheduled for the operation and with the cooperation of the Cardio Vascular Technologist (CVT), Anaesthesiologist, and the OR team, stent placement was done successfully. Fiberoptic bronchoscope was first used to suction secretions and help clear the airway then fluoroscopy to assess the location, and measure the size of oesophageal mass. Electrocautery was done to stop any source of bleeding followed by the rigid brochoscopy for stent placement. Post procedure, the patient was intubated and was admitted to the Intensive Care Unit (ICU) for continuous monitoring. No hemoptysis, vomiting, or untoward complications were noted post stent placement.

\subsection{Case Report \# 4}

A 27-year-old woman who is a foreign health care worker exposed to patients diagnosed of pulmonary tuberculosis, presented productive cough with purulent sputum and right sided chest pain in late 2012. Her Acid Fast Bacilli (AFB) was positive and she was then diagnosed of pulmonary tuberculosis and underwent treatment for 6 months with the following course of drugs: isoniazid, rifampicin, pyrazinamide and ethambutol for 2 months then isoniazid and rifampicin for 4 months. 10 months later she presented stridor, wheezing, shortness of breath and dyspnea. AFB was negative, chest CT scan revealed atelectasis at anterior and superior segment of right upper lobe probably due to mucous impaction or aspergillosis in the bronchi and bronchioles. Bronchoscopy revealed narrowing in the near mid trachea (Figure 7). She was then diagnosed of tracheal stenosis. She was pre- 
scribed with prednisone $5 \mathrm{mg}$ tab and flixotide inhaler.

Two months following her diagnosis, she visited our medical facility for $2^{\text {nd }}$ opinion. She was provided with 2 treatment options. First, was tracheal reconstruction and second was stent placement with the use of silicone stent. Benefits and risks such as infection, bleeding, tracheoesophageal fistula, laryngeal nerve injury, dehiscence of suture line of trachea, restenosis and mortality were discussed. The patient decided to undergo tracheal reconstruction. Neck and chest CT scans revealed tracheal narrowing; $25 \mathrm{~mm}$ long segmental circumferential thickened wall (6 mm thick) at middle portion of trachea (sternal notch level). The narrowest portion has right to left diameter of narrowest portion $=3 \mathrm{~mm}$, anterior-posterior diameter $=6 \mathrm{~mm}$. The right lung presented atelectasis of upper lobe with minimal calcification (Figure 8 and Figure 9). The patient underwent FOB first for direct visualisation before proceeding with the surgery. Tracheal resection and reconstruction under general anaesthesia were done. The stenotic part of trachea (proximal and distal end) was dissected and removed (Figure 10). Post-surgery, she was admitted for close observation and monitoring. No untoward complications were noted and she was maintained on a neck flexion position. Cytopathology report from the tracheal tissue revealed that the trachea was lined by respiratory type mucosa with extensive squamous metaplasia. There were acute and chronic inflammatory infiltrates. The stroma showed fibrosis. There were no granulomas or viral inclusions. No evidence of dysplasia or malignancy noted (Figure 11). The patient was able to recover well following the tracheal reconstruction surgery.

\subsection{Case Report \# 5}

The patient is a 41-year-old female from the Middle East, who presented dyspnea for 2 years. She has underlying condition of bronchial asthma, in which she was taking fluticasone diskus $500 \mathrm{mcg} 2$ times a day and salbutamol Metered Dose Inhaler (MDI) for relief. For the past 2 years she has had frequent hospital admissions due to acute exacerbation of asthma. Chest CT scan revealed the presence of a lobulated well-defined soft tissue density. Endotracheal lesions were seen related to the anterior and right anterolateral walls of the distal trachea just above the level of the carina. It measures about $16 \times 16 \times 19 \mathrm{~mm}$ along its maximum anteroposterior, transverse and craniocaudad dimensions respectively. Bronchoscopy with biopsy was advised for further evaluation. 1
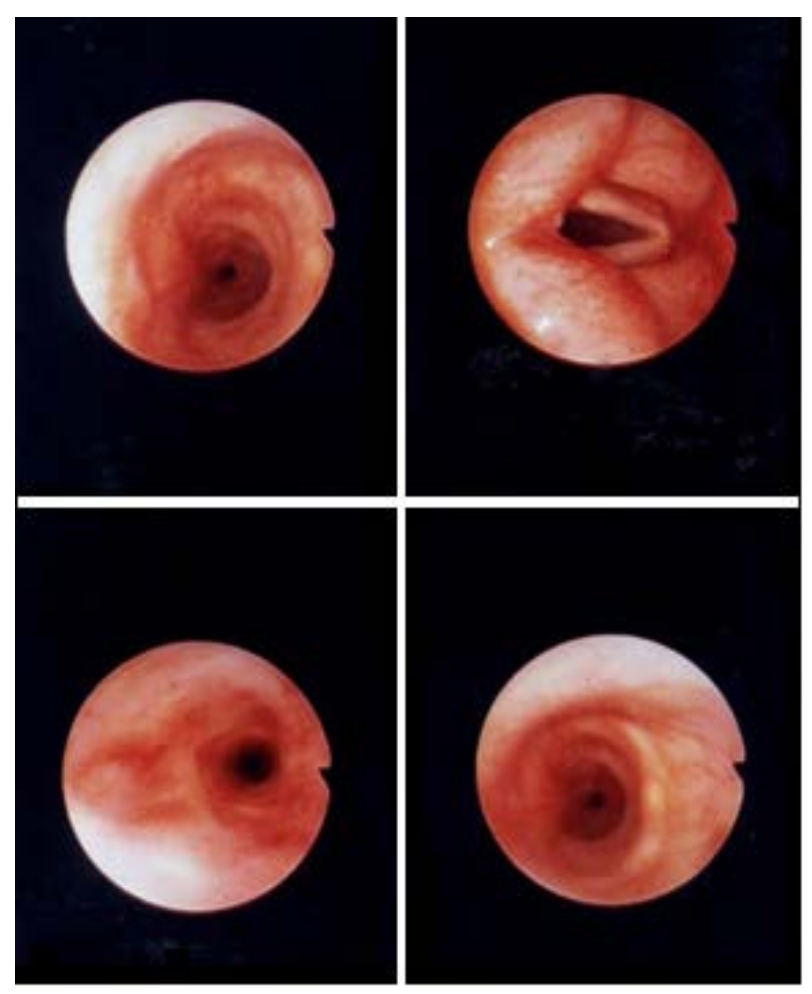

Figure 7. Bronchoscopic findings showed narrowing at the near mid trachea. 


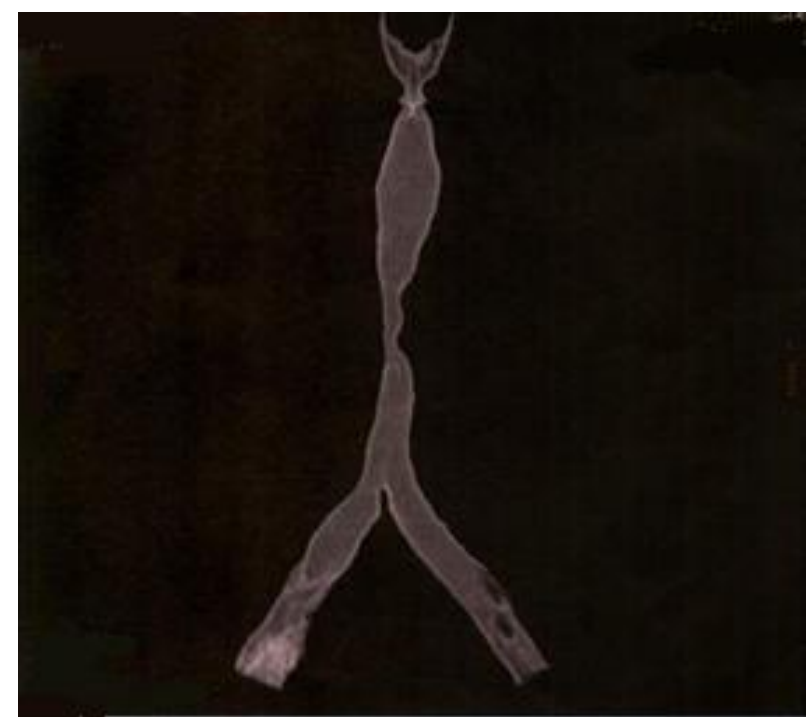

Figure 8. Three-dimensional benign tracheal stenosis.

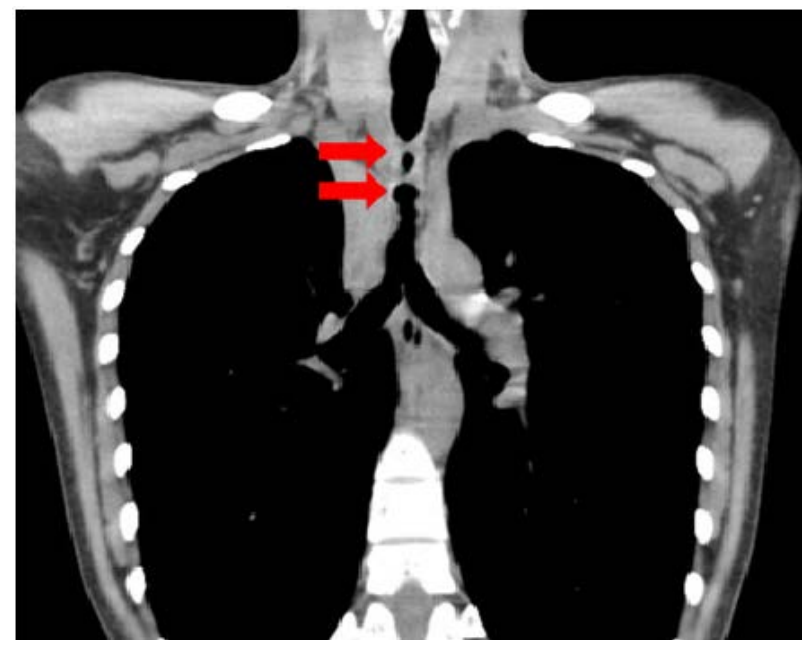

Figure 9. Tracheal narrowing seen in the neck CT scan.

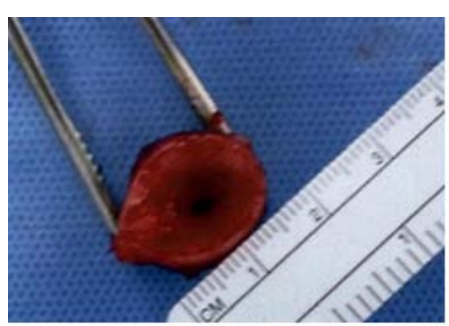

Proximal end

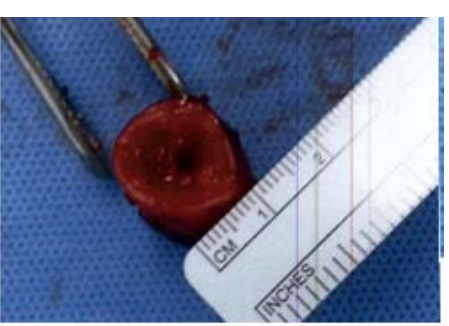

Distal Fnd

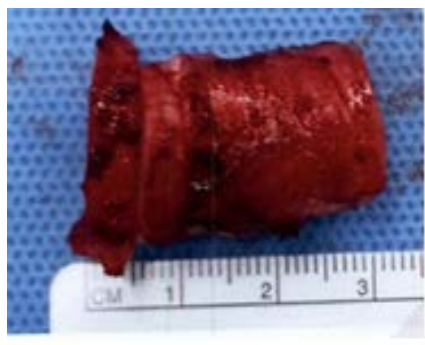

Part of Tracheal Stenosis

Figure 10. The dissected stenotic part of trachea.

month following the recommendation from her local physician, she went for a medical consultation in our facility. Lung Function Test revealed severe obstruction without response to bronchodilator.

FOB was done and revealed mid endotracheal mass (Figure 12). Cytopathology from the biopsy presented bronchial wall with chronic inflammatory cell infiltration without tumour cell or granuloma. Removal of en- 


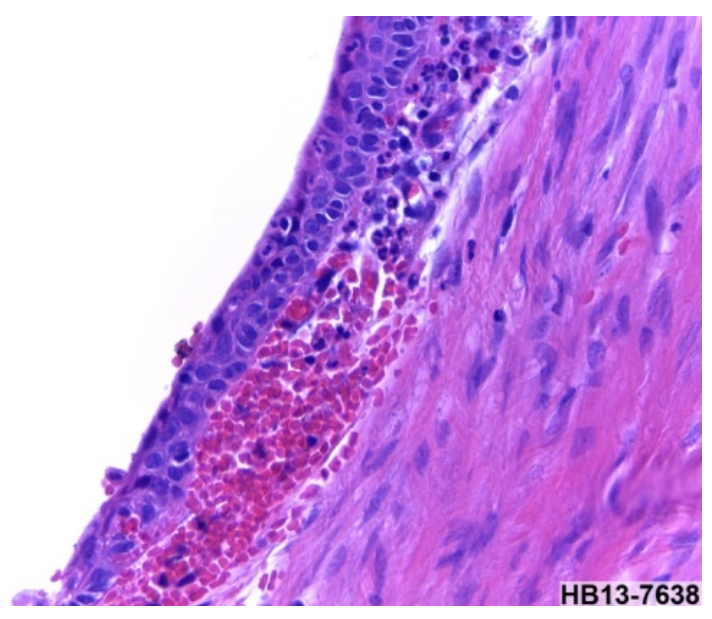

Figure 11. Squamous metaplasia with acute inflammation.

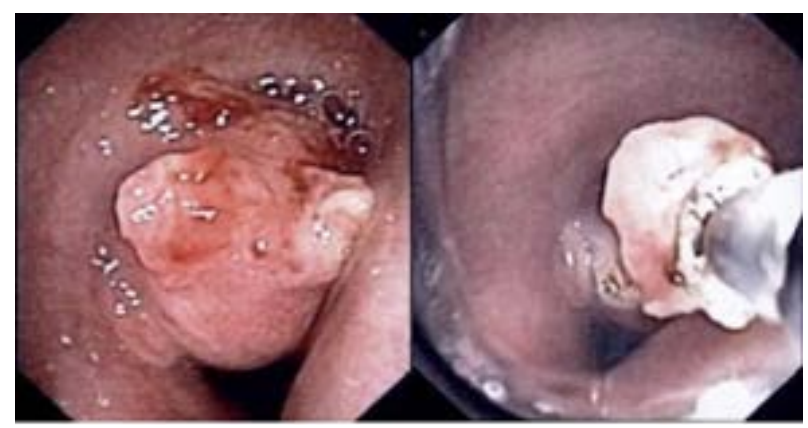

Figure 12. The photo shows the mid endotracheal mass and how it was extracted using bronchoscopy and snare electrocautery.

dotracheal mass then stent placement was advised for management. Benefits and risks such as tracheoesophageal fistula, hypoxia, bleeding and infection were discussed. The endotracheal mass was extracted via the combination of rigid bronchoscope and FOB with snare and electrocautery. She did not manifest untoward complications post procedure. The cytology report from the mass revealed unremarkable covering respiratory epithelial cells, the subepithelial element showed dense infiltration by mostly small lymphoid cells admixed with some plasmacytoid ones, plasma cells, and larger cells. Scattering secondary follicles were noted. There was no granuloma or necrosis.

Tumor panel concluded that the specimens must be sent to the laboratory for the following tests immunohistochemical stains for CD3, CD20, CD5, CD23, CD10, BCL6, BCL2, Ki-67, kappa, lambda, CD43, and cyclin D1 in order to determine if whether this is a lymphoma or a pseudo lymphoma.

\section{Discussion}

\subsection{Tracheal Stenosis Caused by Malignancy}

Both malignant and benign diseases may cause focal narrowing of the tracheobronchial tree. Within the trachea, malignancy predominates [2].

In July 2013, the World Health Organization reported that chronic diseases cause increasing numbers of mortality worldwide. Lung malignancy along with trachea and bronchus cancers caused 1.5 million (2.7\%) deaths in 2011, a remarkable increase from 1.2 million (2.2\%) deaths in 2000. The vast majority of these types of cancers are diagnosed at a critical later stage when the disease has metastasized to other organs and or lymph nodes. Primary tracheal malignancies include squamous cell carcinoma and adenoid cystic carcinoma, among others. Non-small cell lung cancer and small cell lung cancer are obvious causes of obstruction due either to extrinsic 
airway compression, intrinsic airway tumor, or a combination of both [2]. A recent study in 2008 presented that in one series of 264 patients with malignant Esophago-Respiratory Fistulas (ERF), 243 (92\%) had esophageal cancer, 19 (7\%) had lung cancer, and 2 (1\%) had mediastinal tumor [3]. These evidently show that there has been a recorded marked increase in mortality caused by respiratory malignancies specifically cases of airway narrowing or obstruction. In most cases, late assessment and diagnosis are performed, giving the patients only the option of palliative treatment from stent placement. Although there has been several international organizations developing protocols for early diagnosis for lung cancer, it is believed that malignancies causing airway constriction should remain as priority.

Sign and symptoms vary depending on the location of the narrowing and nature of the disease. Patients complain of dyspnea, shortness of breath and dysphagia which is progressive, most of the time constant and unresponsive to bronchodilators. Wheezing maybe present and stridor can be heard especially for severe tracheal obstruction. The onset and progression of these signs and symptoms generally depend on the pathology and extent of the disease. In a study of 207 malignant Trachea-Esophageal Fistulas (TEFs), signs and symptoms include cough in 116 (56\%), aspiration in 77 (37\%), fever in 52 (25\%), dysphagia in 39 (19\%), pneumonia in 11 (5\%), hemoptysis in $10(5 \%)$, and chest pain in 10 (5\%) [4]. Management of airway stenosis especially the use of stents are performed as palliative measures to ease the burden of patients with worsening conditions. Stent placement is less invasive and usually requires IV sedation, giving more opportunity for unstable or critical patients to undergo this technique. On the other hand, surgery or tracheal resection may be more invasive and that requires medical clearance, but still provides permanent remedy. In spite the fact that both methods are proven effective considering both its advantages and disadvantages, it is still imperative that the patient's condition will determine which procedure is deemed appropriate.

Surgical resection remains the gold standard for treatment of airway stenosis. However, most cancer patients are not medically fit and may have been diagnosed on the later stages of malignancy. Palliative measures are offered to relieve worsening symptoms. Treatment includes chemo radiation, brachytherapy, tumor ablation, endoscopic techniques, and stent placements. These options if successfully done may improve breathing, swallowing mechanism and quality of life.

The latest standard therapy for cancer patients with airway stenosis is endoscopic or radiologic placement of endotracheal-covered Self-Expanding Metallic Stents (SEMS) [5]. A covered expandable endotracheal stent (SEMS) can relieve symptoms in more than $80 \%$ of patients with malignant stenosis [6] [7]. If performed early following diagnosis, this treatment may positively change quality of life and improve survival. Endotracheal stents can be inserted with the patient under general anesthesia via rigid or flexible bronchoscope with moderate sedation. The mean survival period of patients with ERF was 2.8 months in one study, but in patients with esophageal stents it was 3.4 months. With only supportive therapy, it was 1.3 months [3]. Overall median survival times after diagnosis of ERF is only 8 weeks [8]. Patients generally tolerate stent placement but close monitoring is needed so to assess complications ahead of time and manage appropriately. Potential issues include recurrence of obstruction, growth of granulation tissue, stent occlusion and migration, bleeding and infection.

A recent study by Homann et al. reported late complications in 71 of 133 stented patients (53.4\%) with a quarter of patients experiencing several complications. Recurrent dysphagia connected to tumor ingrowth (22\%), overgrowth (15\%), stent migration (9\%), and food bolus obstruction (21\%) were the most common complications, followed by the development of esophago-airway fistulas (9\%). Successfully retreated patients had a markedly longer life expectancy compared to those who did not come back for further management. In an additional retrospective review of 97 patients with SEMS placement, dysphagia improved in $86 \%$ and tracheosophageal fistula symptoms in $90 \%$ of the patients [9].

\subsection{Benign Cause}

A variable degree of stenosis has been reported in up to $90 \%$ of patients with tuberculosis [10]. The application of surgical resection to airway narrowing caused by benign disease requires meticulous cooperation with the anesthesiologist, pulmonologist, nursing staff and thoracic surgeon who have been well trained in the reconstruction of complicated tracheobronchial abnormalities. Surgery can be done for benign stenosis that affects less than half of the trachea. The type of surgery performed depends on the site of stenosis. Preoperative requirements include history, physical examination, radiographic imaging such as chest X-rays, CT scans and bronchoscopy results. The direct visualization provided by the bronchoscope will show the anatomy of the air- 
way at the same time the affected parts of the stenosis. It is important to note the distance from the stenosis to anatomic landmarks in addition to the length of the stenotic segment and the status of the mucosa. Operation of the airway needs constant communication between the pulmonologist, surgeon and anesthesiologist. The steps of tracheal resection and reconstruction are the following: 1) localize the diseased segment, 2) mobilize the trachea, 3) transect the trachea, 4) resect the affected area, 5) and reconstruct [11]. Anastomotic complications are uncommon post operatively but may lead to serious complications. In one study conducted by Wright et al. of 853 patients, 95\% had a good result and 4\% had an airway maintained by tracheostomy or T-tube. Tracheal resection with reanastomosis is seen as a procedure of choice given its high success rate (71\% - 95\%) and minimal morbidity [12]. It is usually successful and patients often fully recover from the operation. Although more invasive that bronchoscopy, tracheal resection has been proven to be effective in management of airway narrowing given the patient is stable and in the hands of a well-trained specialist.

\section{Conclusion}

Central airway stenosis is a life-threatening condition with severe pulmonary complications maybe caused by several factors. Patients diagnosed of airway constriction or obstruction often present with comorbidities that require medical attention. There are a number of factors considered in order to choose the appropriate treatment for the patient. These are nature of the disease, urgency for treatment, alternative procedure, current health status and improvement on the quality of life of the patients. Treatment is individualized and the management of this condition requires technical expertise of thoracic surgeons, pulmonologists and anesthesiologists. If caught early and treatment has been determined and planned following diagnosis, improvement on the quality of life and potentially survival, may be achieved.

\section{Acknowledgements}

With immense gratitude, we would like to acknowledge the assistance of Dr. Chirotchana Suchato, Dr. Rergchai Varatorn and Dr. Chaiyos Kunanusont for their encouragement and assistance. We also wish to thank the staff of Bangkok Hospital Group; Bangkok Chest and Respiratory Center, Operating room complex and Bangkok Research and Development office for their unending support. Lastly, we thank the coauthors whose work has greatly contributed in the completion of this research paper.

\section{References}

[1] Homann, N., Noftz, M.R., Klingenberg-Noftz, R.D., et al. (2008) Delayed Complications after Placement of Self-Expanding Stents in Malignant Esophageal Obstruction: Treatment Strategies and Survival Rate. Digestive Diseases and Sciences, 53, 334-340. http://dx.doi.org/10.1007/s10620-007-9862-9

[2] Grenier, P.A., Beigelman-Aubry, C. and Brillet, P.Y. (2009) Nonneoplastic Tracheal and Bronchial Stenosis. Radiology Clinic North America, 47, 243-260. http://dx.doi.org/10.1016/j.rcl.2008.11.011

[3] Balazas, A., Kupcsulik, P.K. and Galambos, Z. (2008) Esophagorespiratory Fistiluas of Timorous Origin. Non-Operative Management of 264 Cases in a 20 Year Period. European Journal Cardio-Thoracic Surgery, 34, 1103-1107. http://dx.doi.org/10.1016/j.ejcts.2008.06.025

[4] Burt, M., Diehl, W., Martini, N., et al. (1991) Malignant Esophagorespiratory Fistula: Management Options and Survival. Annals of Thoracic Surgery, 52, 1222-1229. http://dx.doi.org/10.1016/0003-4975(91)90005-B

[5] Reed, M.F. and Mathisen, D.J. (2003) Tracheoesophageal Fistula. Chest Surgery Clinics of North America, 13, 271289. http://dx.doi.org/10.1016/S1052-3359(03)00030-9

[6] Rodriguez, A.N. and Diaz-Jimenez, J.P. (2012) Malignant Respiratory-Digestive Fistulas. Current Opinion in Pulmonary Medicine, 16, 329-333. http://dx.doi.org/10.1097/MCP.0b013e3283390de8

[7] Shin, J.H., Song, H.Y., Ko, G.Y., et al. (2004) Esophagorespiratory Fistula: Long Term Results of Palliative Treatment with Covered Expandable Metallic Stents in 61 Patients. Radiology, 232, 252-259. http://dx.doi.org/10.1148/radiol.2321030733

[8] Choi, M.K., Park, Y.H., Hong, J.Y., et al. (2010) Clinical Implications of Esophagorespiratory Fistulae in Patients with Esophageal Squamous Cell Carcinoma (SCCA). Medical Oncology, 27, 1234-1238. http://dx.doi.org/10.1007/s12032-009-9364-z

[9] Puchalski, J. and Musani, A. (2013) Tracheobronchial Stenosis Causes and Advances in Management. Clinics in Chest Medicine, 34, 557-567. 
[10] Yamamoto, K., Kojima, F., Tomiyama, K., et al. (2011) Meta-Analysis of Therapeutic Procedures for Acquired Subglottic Stenosis in Adults. Annals of Thoracic Surgery, 91, 1747-1753. http://dx.doi.org/10.1016/j.athoracsur.2011.02.071

[11] Cameronm, J., Cameron, A., Su, S. and Cooper, J. (2011) Chest Wall, Mediastinum, and Trachea. In: Current Surgical Therapy, 10th Edition, 684-689. http://www.expertconsultbook.com

[12] Shiraishi, T., Yanagisawa, J., Higuchi, T., et al. (2011) Tracheal Resection for Malignant and Benign Diseases: Surgical Results and Perioperative Considerations. Surgery Today, 41, 490-495. http://dx.doi.org/10.1007/s00595-010-4303-3 
Scientific Research Publishing (SCIRP) is one of the largest Open Access journal publishers. It is currently publishing more than 200 open access, online, peer-reviewed journals covering a wide range of academic disciplines. SCIRP serves the worldwide academic communities and contributes to the progress and application of science with its publication.

Other selected journals from SCIRP are listed as below. Submit your manuscript to us via either submit@scirp.org or Online Submission Portal.
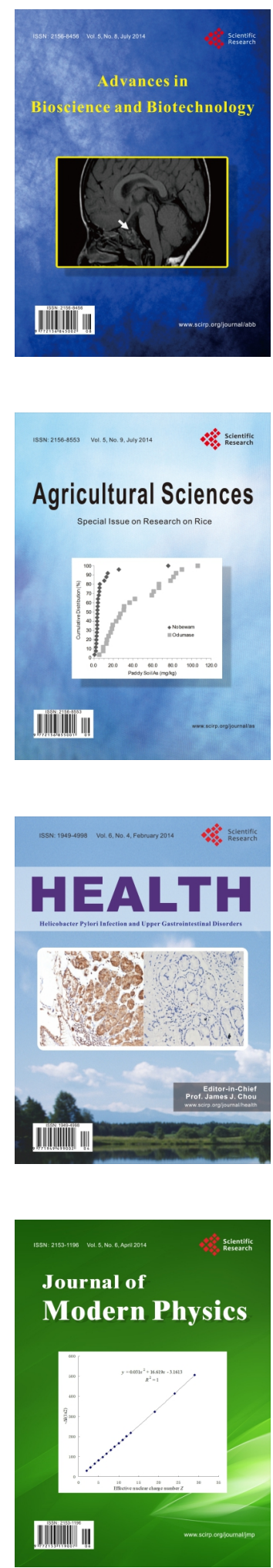
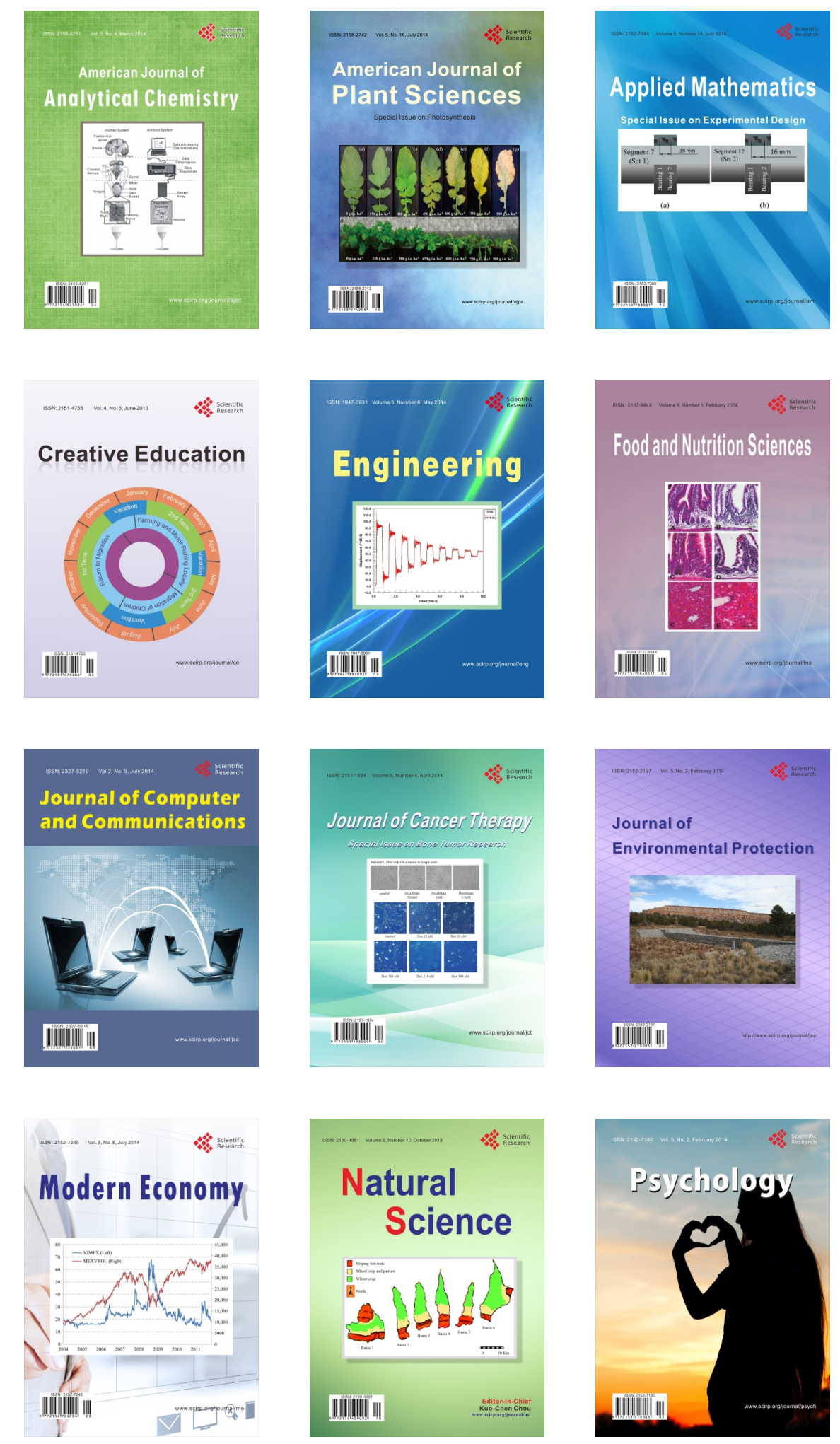